\title{
Computation of several power indices by generating functions
}

\author{
J.M. Alonso-Meijide ${ }^{\mathrm{a}, 1}$, J. Freixas ${ }^{\mathrm{b}, 2,4}, \mathrm{X}$. Molinero ${ }^{\mathrm{b}, 3,4, *}$ \\ ${ }^{a}$ Department of Statistics and Operations Research and Faculty of Sciences of Lugo, \\ University of Santiago de Compostela (Spain). \\ ${ }^{b}$ Department of Applied Mathematics III and High Engineering School (Manresa Campus), \\ Technical University of Catalonia (Spain).
}

\begin{abstract}
In this paper we propose methods to compute the Deegan-Packel, the Public Good, and the Shift power indices by generating functions for the particular case of weighted voting games. Furthermore, we define a new power index which combines the ideas of the Shift and the Deegan-Packel power indices and also propose a method to compute it with generating functions. We conclude by some comments about the complexity to compute these power indices.
\end{abstract}

Keywords: Simple games, Weighted voting games, Power indices, Generating functions.

\section{Introduction}

Generating functions are used in several operational research problems to construct counting algorithms $[13,38,30]$. The flexibility of the generating functions allows the development of finite algorithms for problems related to inclusion and exclusion questions as, e.g., optimal allocation [27, 24], discrete optimization [40,11,36], production control [18], weighted voting systems [28, $26,22,23,25]$ or computation of power indices for weighted majority games $[8$, $1]$.

The classical power indices suggested in order to assess the a priori distribution of power in a simple game include the Shapley-Shubik [34, 33], the Banzhaf

\footnotetext{
*corresponding author

Email addresses: josemaria.alonso@usc.es (J.M. Alonso-Meijide),

josep.freixas@upc.edu (J. Freixas), xavier.molinero@upc.edu (X. Molinero)

${ }^{1}$ Research partially supported by Grants INCITE09-207-064-PR from the Xunta de Galicia, ECO2008-03484-C02-02/ECON of the Spanish Science and Innovation Ministry and the European Regional Development Fund, and MTM 2011-27731-C03-03.

${ }^{2}$ Research partially funded by Grant SGR 2009-1029 of Generalitat de Catalunya

${ }^{3}$ Research partially funded by Grant SGR 2009-1137 of Generalitat de Catalunya

${ }^{4}$ Research partially funded by Grants MTM 2009-08037 and MTM 2012-34426 from the Spanish Economy and Competitiveness Ministry.
} 
[6], the Johnston [19], the Deegan-Packel [12], and the Public Good [15] power indices. This is not a comprehensive list and other power indices could be listed; we just mention here one more power index, the Shift power index introduced in Alonso-Meijide and Freixas [4], since we will deal with a slight variation of it (the Shift Deegan-Packel power index) in this paper.

One of the main difficulties with these indices is that computation generally requires the sum of a very large number of terms. Owen in [31] introduces the multilinear extension of an $n$-person game as a help in computing the power indices of large games, and gives a generalization of the Shapley-Shubik power index. Owen in [32] also shows that the Banzhaf power index can be obtained by differentiating the multilinear extension of a game. Later, Alonso-Meijide et al. [3] presents again the multilinear extension to compute the Johnston, the Deegan-Packel and the Public Good power indices.

A different useful procedure to compute power indices is provided by the generating functions techniques. The main objective of this paper is to analyze whether some modification of the generating function techniques might be used to calculate the Deegan-Packel, the Public Good, and the Shift power indices for weighted voting games. These three indices are defined on the basis of either minimal winning coalitions (in the case of the Deegan-Packel and the Public Good indices) or shift-minimal winning coalitions (in the case of the Shift power index) [4]. Specially in games with a large number of players, it is not known a priori which coalitions are minimal winning or shift-minimal winning.

The advantage of the procedures presented here is that once the corresponding generating function has been defined, we provide an algorithm to easily compute these indices. Although some techniques on generating functions have been applied to computing (the Shapley-Shubik, the Banzhaf-Coleman and the Owen) power indices $[1,21,7]$, now we complete such analysis and provide new methods, based again on generating functions, to compute the Deegan-Packel, the Public Good, and the Shift power indices.

The paper is organized as follows. Section 2 is devoted to reviewing the main power indices and to introducing a new power index. In Section 3, we recall generating functions and present new methods based on them to compute the Public Good, the Deegan-Packel, the Shift and the Shift Deegan-Packel power indices by means of generating functions. In section 4 we provide the required complexity for our methods. Conclusion and future research end the paper in Section 5 .

\section{Preliminaries: Simple games and power indices}

A simple game consists of a finite set of players $N$ and a set of winning coalitions, $\mathcal{W}$, which is a collection of subsets of $N$ with the following three properties: $\emptyset \notin \mathcal{W} ; N \in \mathcal{W}$; and monotonicity: If $S \in \mathcal{W}$ and $S \subseteq T$, then $T \in \mathcal{W}$

Thus, either the family of winning coalitions $\mathcal{W}$ or the subfamily of minimal winning coalitions $\mathcal{W}^{m}=\{S \in \mathcal{W}: T \subseteq S \Rightarrow T \notin \mathcal{W}\}$ with the inclusion determines the game. 
For the interested reader, there are some applications and specific studies about simple games in $[20,17,39,35]$.

The class of weighted voting games is probably the most important subfamily of simple games since many real-world examples are of this type. Generating functions apply precisely to the class of weighted voting games and therefore constitute a meaningful tool to compute power indices for these games. As far as we know this technique has only been applied for the Banzhaf (and Coleman), the Shapley-Shubik and the Owen power indices $[1,21,7]$. The main goal of the paper is to provide a systematic symbolic approach to compute four alternative significant power indices. Our power indices will be defined from weighted voting games. $(N, \mathcal{W})$ is called a weighted voting game if there exist natural integers $w_{1}, \ldots, w_{n}$ such that every coalition $S$ verifies $S \in \mathcal{W}$ if and only if the sum of the $w_{i}$ 's, $i \in S$, is at least equal to some preset quota $q$, i.e., $\sum_{i \in S} w_{i} \geq q$.

The number $w_{i}$ is interpreted as the number of votes that player $i$ owns, and $q$ is the least total number of votes necessary to pass a decision. Such representation for $(N, \mathcal{W})$ is indicated by $\left[q ; w_{1}, w_{2}, \ldots, w_{n}\right]$, where it is assumed that $w_{1} \geq w_{2} \geq \cdots \geq w_{n}$, and $w(S)$ stands for $\sum_{i \in S} w_{i}$. Note that for $n \geq 4$ there are simple games which are not weighted, but every simple game can be written as an intersection of weighted voting games.

Loosely speaking, a power index is a function $g$ which assigns to a simple game $(N, \mathcal{W})$ a vector $g(N, \mathcal{W}) \in \mathbb{R}^{n}$ where each component $g_{i}(N, \mathcal{W})$ is a measure for the $i$ th player in the simple game $(N, \mathcal{W})$ according to $g$. As $N$ does not change in the rest of the paper, we will write $g(\mathcal{W})$ instead of $g(N, \mathcal{W})$ hereafter. Table 1 shows known definitions of power indices (see e.g. [14] for a comprehensive treatment on power indices) that will be considered in this work. In this table we use a known notation: Given a simple game $(N, \mathcal{W})$, we define a swing for player $i$ by a coalition $S \subseteq N \backslash\{i\}$ such that $S \notin \mathcal{W}$ but $S \cup\{i\} \in \mathcal{W}$, and we denote $\eta_{i}(\mathcal{W})$ the number of swings for player $i \in N$. We also define $s=|S|$ and $\mathcal{C}_{i}=\{S \subseteq N \backslash\{i\}: S \notin \mathcal{W} \wedge S \cup\{i\} \in \mathcal{W}\}$ so that $\eta_{i}(\mathcal{W})=\left|\mathcal{C}_{i}\right|$. Let $\mathcal{W}_{i}^{m}=\left\{S \in \mathcal{W}^{m}: i \in S\right\}$; and let $c_{i}(\mathcal{W})=\left|\mathcal{W}_{i}^{m}\right|$. To define the Shift power index [4], it is necessary to introduce the desirability relation defined in [16].

Let $(N, \mathcal{W})$ be a simple game, $i$ and $j$ be two players. Players $i$ and $j$ are said to be equally desirable, denoted by $i \sim j$, if for any coalition $S \in N \backslash\{i, j\}$, then $S \cup\{i\} \in \mathcal{W} \Leftrightarrow S \cup\{j\} \in \mathcal{W}$. Let $(N, \mathcal{W})$ be a simple game, $i$ and $j$ be two voters, then player $i$ is said to be (strictly) more desirable than $j$, denoted by $i \succ j$, if the following two conditions are fulfilled:

1. $\forall S \in N \backslash\{i, j\}$, then $S \cup\{j\} \in \mathcal{W} \Rightarrow S \cup\{i\} \in \mathcal{W}$.

2. $\exists T \in N \backslash\{i, j\}$ such that $T \cup\{i\} \in \mathcal{W}$ and $T \cup\{j\} \notin \mathcal{W}$.

Let again $(N, \mathcal{W})$ be a simple game, $i$ and $j$ be two voters, then player $i$ is said to be at least as desirable as $j$ (as a coalitional partner), denoted by $i \succsim j$, if $i \succ j$ or $i \sim j$. The $\succsim$ relation is known as the desirability relation.

Now, we go in deep into the notion of shift minimal winning coalitions. See [35] for references and history on the mathematical use of the shift ordering. 


\begin{tabular}{ccc} 
Power Index & Definition & References \\
\hline Banzhaf relative & $\beta_{i}(\mathcal{W})=\frac{\eta_{i}(\mathcal{W})}{\sum_{j=1}^{n} \eta_{j}(\mathcal{W})}$ & {$[6]$} \\
Shapley-Shubik & $\varphi_{i}(\mathcal{W})=\sum_{S \in \mathcal{C}_{i}} \frac{s !(n-s-1) !}{n !}$ & {$[34]$} \\
Deegan-Packel & $\rho_{i}(\mathcal{W})=\frac{1}{\left|\mathcal{W}^{m}\right|} \sum_{S \in \mathcal{W}_{i}^{m}} \frac{1}{s}$ & {$[12]$} \\
Public Good & $h_{i}(\mathcal{W})=\frac{c_{i}(\mathcal{W})}{\sum_{j=1}^{n} c_{j}(\mathcal{W})}$ & {$[15]$} \\
Shift & $f_{i}(\mathcal{W})=\frac{s_{i}(\mathcal{W})}{\sum_{j=1}^{n} s_{j}(\mathcal{W})}$
\end{tabular}

Table 1: Some known power indices.

Let $(N, \mathcal{W})$ be a simple game and $\succsim$ be its desirability relation. A coalition $S \in \mathcal{W}^{m}$ is shift minimal if for every $i \in S$ and $j \notin S$ such that $i \succ j$ it holds $(S \backslash\{i\}) \cup\{j\} \notin \mathcal{W}$. From now on, the set of shift minimal winning coalitions will be denoted by $\mathcal{W}^{s}$, i.e.,

$$
\left.\mathcal{W}^{s}=\left\{S \in \mathcal{W}^{m}: \forall(i \in S \wedge j \notin S: i \succ j) \Rightarrow(S \backslash\{i\}) \cup\{j\} \notin \mathcal{W}\right)\right\} .
$$

Moreover, we denote $s_{i}(\mathcal{W})=\left|\mathcal{W}_{i}^{s}\right|$, where $\mathcal{W}_{i}^{s}=\left\{S \in \mathcal{W}^{s}: i \in S\right\}$.

Finally, we introduce another power index which combines the ideas of the Shift and the Deegan-Packel indices, as far as we know this is a new power index. Let $(N, \mathcal{W})$ be a simple game, the Shift Deegan-Packel index for each player $i \in N$ is the real number:

$$
\mu_{i}(\mathcal{W})=\frac{1}{\left|\mathcal{W}^{s}\right|} \sum_{S \in \mathcal{W}_{i}^{s}} \frac{1}{s} \text { so that } \sum_{i=1}^{n} \mu_{i}(\mathcal{W})=1
$$

It assumes that:

1. Only shift minimal winning coalitions will emerge victorious,

2. Each shift minimal winning coalition has an equal probability of forming, and

3. Players in a shift minimal winning coalition divide the "spoils" equally.

The Shift-Deegan Packel index is an intermediate solution between the DeeganPackel index and the Shift power index. In its definition, only shift minimal winning coalitions appear, but the matter may be approached differently than the Shift index, because the size of the coalitions is taken into account. The 
involved coalitions in the computation of the Shift Deegan-Packel index and the Shift index are the same, and the number of these involved coalitions is inferior to that of Deegan-Packel and Public Good indices.

\section{Generating functions to compute the Deegan-Packel, Public Good, Shift and Shift Deegan-Packel power indices}

In general, the computation of the previous indices needs a great number of operations. Generating functions $[13,38,30]$ give us a useful method to count the number of elements $c(r)$ of a finite set, where these elements have a configuration that depends on a characteristic $r$. An application of these functions in the field of simple games allows to recover the number of possible coalitions of a given kind from the set of its coefficients, while the voting power of the coalition can easily be read looking at its set of exponents. Brams and Affuso [9] provide an example of a generating function for the classical power indices.

The generating function of the sequence of numbers $\left[a_{0}, a_{1}, a_{2}, \ldots\right]$ is the formal series $F(t)=\sum_{i \geq 0} a_{i} t^{i}$, and can be finite or infinite. The variable $t$ serves to identify $a_{i}$ as the coefficient of $t^{i}$ in $F(t)$.

Example 1. Consider the series

$$
\prod_{r=1}^{n}\left(1+x_{r} t\right)=\sum_{r=0}^{n} a_{r} t^{r},
$$

where $a_{0}=1$ and for $r>0, a_{r}$ is given by

$$
a_{r}=\sum_{1 \leq i_{1}<i_{2}<\ldots<i_{r} \leq n} x_{i_{1}} x_{i_{2}} \ldots x_{i_{r}} .
$$

The coefficients $a_{r}$ are symmetric functions on the variables $x_{1}, x_{2}, \ldots, x_{n}$. The number of terms of the coefficient $a_{r}$ coincides with the number of combinations of $r$ elements of a finite set formed by $n$ elements. If all the values $x_{r}$ are 1 it holds that

$$
(1+t)^{n}=\sum_{r=0}^{n}\left(\begin{array}{l}
n \\
r
\end{array}\right) t^{r},
$$

because, in this case the coefficients $a_{r}$ are the number of combinations of $r$ elements of a finite set formed by $n$ elements. Then, the function $F(t)=$ $(1+t)^{n}$ is the generating function of the numbers $a_{r}=\left\{\left(\begin{array}{l}n \\ r\end{array}\right): r=0,1, \ldots, n\right\}$. That is, the binomial coefficients $\left(\begin{array}{l}n \\ r\end{array}\right)$ can be obtained by means of the function $(1+t)^{n}$ because they coincide with the coefficients of the formal series.

Next, we will use generating functions of several variables, for example

$$
F(x, y, z)=\sum_{k \geq 0} \sum_{j \geq 0} \sum_{l \geq 0} a_{k j l} x^{k} y^{j} z^{l}
$$


where $a_{k j l}$ are real numbers depending on $k, j$ and $l$.

Cantor [10] (and Lucas [29]) used this method to compute the ShapleyShubik index and Brams and Affuso [9] used the same method to compute the Banzhaf index. In $[21,7]$ generating functions are used to compute power indices in games with restricted situations and in games with multiple majority. Alonso-Meijide and Bowles [2] used the same method to compute power indices in games endowed with a coalition structure and presented an application to the International Monetary Fund. Alonso-Meijide et al. [1] also used the generating functions to compute power indices in multiple majority games with a coalition structure and also presented an application to the European Union. In all these papers, the power indices are either the Banzhaf or the Shapley-Shubik ones (or modifications of them). To our knowledge, the method of generating functions is only used to compute indices based on the number of swings. In this paper, we present a result that allows the computation, with generating functions, of indices not based on the number of swings but based on minimal (or shift-minimal) winning coalitions.

\subsection{Deegan-Packel and Public Good power indices}

In this section we provide a method to compute Deegan-Packel and Public Good power indices by means of the generating function in a weighted voting game. As far as we know this is a new procedure.

To compute the Deegan-Packel index in a weighted voting game, first, we will employ a polynomial that has as many addends as coalitions can be formed, i.e., $2^{n}$ coalitions. Among them, we will retain the addends that correspond to the winning coalitions, and, finally, among them, we will only keep the monomials that correspond to minimal winning coalitions. To obtain this polynomial, we need to employ $n+2$ variables, one for each player, another to indicate the number of players in each coalition and, finally, a variable that indicates the weight of the coalition.

Let $(N, \mathcal{W})$ be a simple game, and let $m_{k}^{i}(\mathcal{W})$ be the number of minimal winning coalitions with cardinality $k$ which the player $i$ belongs to. Then the Deegan-Packel index for player $i \in N$ can be expressed as (see previous definition in Table 1):

$$
\rho_{i}(\mathcal{W})=\frac{1}{\left|\mathcal{W}^{m}\right|} \sum_{S \in \mathcal{W}_{i}^{m}} \frac{1}{s}=\frac{1}{\left|\mathcal{W}^{m}\right|} \sum_{k=1}^{n} \frac{m_{k}^{i}(\mathcal{W})}{k}
$$

Now, given a weighted voting game $(N, \mathcal{W})$ with representation $\left[q ; w_{1}, w_{2}, \ldots, w_{n}\right]$, we establish three steps to compute $m_{k}^{i}(\mathcal{W})$. In the first step, we consider the generating function given by

$$
S\left(x, z_{1}, z_{2}, \ldots z_{n}, t\right):=\prod_{k=1}^{n}\left(1+x^{w_{k}} z_{k} t\right) .
$$


The corresponding coefficient $c_{k, j}^{i_{1}, \ldots, i_{k}}$ of $S\left(x, z_{1}, z_{2}, \ldots z_{n}, t\right)$,

$$
S\left(x, z_{1}, z_{2}, \ldots z_{n}, t\right)=\sum_{k, j \geq 1} c_{k, j}^{i_{1}, \ldots, i_{k}} x^{j} t^{k} z_{i_{1}} \ldots z_{i_{k}}
$$

is the number of coalitions with players $i_{1}, \ldots, i_{k}$ (coalition with cardinality $k$ ) whose weights add $j$ (i.e., $w_{i_{1}}+\ldots+w_{i_{k}}=j$ ).

In the second step, we remove those monomials in which the power of $x$ is less than $q$, that is, we only keep winning coalitions. In the last third step, we remove those monomials which are divisible by another monomial of the function, that is, we only keep minimal winning coalitions. The total number of terms of this function coincides with the number of minimal winning coalitions of the game, $\left|\mathcal{W}^{m}\right|$.

Finally, to obtain the number $m_{k}^{i}(\mathcal{W})$, we only need to select those terms of the previous function in which the variable $z_{i}$ appears. The power of the variable $t$ indicates the number of players of this minimal winning coalition.

Below we present an example to compute the Deegan-Packel index.

Example 2. Consider the weighted voting game $(N, \mathcal{W})$ with representation

$$
[30 ; 28,16,5,4,3,3] \text {. }
$$

In a first step, we compute the function $S\left(x, z_{1}, z_{2}, \ldots, z_{6}, t\right)$

$$
\begin{aligned}
& S\left(x, z_{1}, z_{2}, \ldots, z_{6}, t\right)=\prod_{k=1}^{n}\left(1+x^{w_{k}} z_{k} t\right)= \\
& \left(1+x^{28} z_{1} t\right)\left(1+x^{16} z_{2} t\right)\left(1+x^{5} z_{3} t\right)\left(1+x^{4} z_{4} t\right)\left(1+x^{3} z_{5} t\right)\left(1+x^{3} z_{6} t\right)= \\
& x^{59} z_{1} z_{2} z_{3} z_{4} z_{5} z_{6} t^{6}+x^{31} z_{2} z_{3} z_{4} z_{5} z_{6} t^{5}+x^{43} z_{1} z_{3} z_{4} z_{5} z_{6} t^{5}+x^{54} z_{1} z_{2} z_{4} z_{5} z_{6} t^{5}+ \\
& x^{55} z_{1} z_{2} z_{3} z_{5} z_{6} t^{5}+x^{56} z_{1} z_{2} z_{3} z_{4} z_{6} t^{5}+x^{56} z_{1} z_{2} z_{3} z_{4} z_{5} t^{5}+x^{15} z_{3} z_{4} z_{5} z_{6} t^{4}+ \\
& x^{26} z_{2} z_{4} z_{5} z_{6} t^{4}+x^{27} z_{2} z_{3} z_{5} z_{6} t^{4}+x^{28} z_{2} z_{3} z_{4} z_{6} t^{4}+x^{28} z_{2} z_{3} z_{4} z_{5} t^{4}+x^{38} z_{1} z_{4} z_{5} z_{6} t^{4}+ \\
& x^{39} z_{1} z_{3} z_{5} z_{6} t^{4}+x^{40} z_{1} z_{3} z_{4} z_{6} t^{4}+x^{40} z_{1} z_{3} z_{4} z_{5} t^{4}+x^{50} z_{1} z_{2} z_{5} z_{6} t^{4}+x^{51} z_{1} z_{2} z_{4} z_{6} t^{4}+ \\
& x^{51} z_{1} z_{2} z_{4} z_{5} t^{4}+x^{52} z_{1} z_{2} z_{3} z_{6} t^{4}+x^{52} z_{1} z_{2} z_{3} z_{5} t^{4}+x^{53} z_{1} z_{2} z_{3} z_{4} t^{4}+x^{10} z_{4} z_{5} z_{6} t^{3}+ \\
& x^{11} z_{3} z_{5} z_{6} t^{3}+x^{11} z_{3} z_{4} z_{6} t^{3}+x^{12} z_{3} z_{4} z_{5} t^{3}+x^{22} z_{2} z_{5} z_{6} t^{3}+x^{23} z_{2} z_{4} z_{6} t^{3}+ \\
& x^{23} z_{2} z_{4} z_{5} t^{3}+x^{24} z_{2} z_{3} z_{6} t^{3}+x^{24} z_{2} z_{3} z_{5} t^{3}+x^{25} z_{2} z_{3} z_{4} t^{3}+x^{34} z_{1} z_{5} z_{6} t^{3}+ \\
& x^{35} z_{1} z_{4} z_{6} t^{3}+x^{35} z_{1} z_{4} z_{5} t^{3}+x^{36} z_{1} z_{3} z_{6} t^{3}+x^{36} z_{1} z_{3} z_{5} t^{3}+x^{37} z_{1} z_{3} z_{4} t^{3}+ \\
& x^{47} z_{1} z_{2} z_{6} t^{3}+x^{47} z_{1} z_{2} z_{5} t^{3}+x^{48} z_{1} z_{2} z_{4} t^{3}+x^{49} z_{1} z_{2} z_{3} t^{3}+ \\
& x^{44} z_{1} z_{2} t^{2}+x^{33} z_{1} z_{3} t^{2}+x^{32} z_{1} z_{4} t^{2}+x^{31} z_{1} z_{5} t^{2}+x^{31} z_{1} z_{6} t^{2}+ \\
& x^{21} z_{2} z_{3} t^{2}+x^{20} z_{2} z_{4} t^{2}+x^{19} z_{2} z_{5} t^{2}+x^{19} z_{2} z_{6} t^{2}+x^{9} z_{3} z_{4} t^{2}+ \\
& x^{8} z_{3} z_{5} t^{2}+x^{8} z_{3} z_{6} t^{2}+x^{7} z_{4} z_{5} t^{2}+x^{7} z_{4} z_{6} t^{2}+x^{6} z_{5} z_{6} t^{2}+
\end{aligned}
$$




$$
x^{28} z_{1} t+x^{16} z_{2} t+x^{5} z_{3} t+x^{4} z_{4} t+x^{3} z_{5} t+x^{3} z_{6} t+1 .
$$

In a second step, we choose those terms in which the power of $x$ is greater than or equal to 30 , that is,

$$
\begin{gathered}
x^{59} z_{1} z_{2} z_{3} z_{4} z_{5} z_{6} t^{6}+x^{31} z_{2} z_{3} z_{4} z_{5} z_{6} t^{5}+x^{43} z_{1} z_{3} z_{4} z_{5} z_{6} t^{5}+ \\
x^{54} z_{1} z_{2} z_{4} z_{5} z_{6} t^{5}+x^{55} z_{1} z_{2} z_{3} z_{5} z_{6} t^{5}+x^{56} z_{1} z_{2} z_{3} z_{4} z_{6} t^{5}+x^{56} z_{1} z_{2} z_{3} z_{4} z_{5} t^{5}+ \\
x^{38} z_{1} z_{4} z_{5} z_{6} t^{4}+x^{39} z_{1} z_{3} z_{5} z_{6} t^{4}+x^{40} z_{1} z_{3} z_{4} z_{6} t^{4}+x^{40} z_{1} z_{3} z_{4} z_{5} t^{4}+ \\
x^{50} z_{1} z_{2} z_{5} z_{6} t^{4}+x^{51} z_{1} z_{2} z_{4} z_{6} t^{4}+x^{51} z_{1} z_{2} z_{4} z_{5} t^{4}+x^{52} z_{1} z_{2} z_{3} z_{6} t^{4}+ \\
x^{52} z_{1} z_{2} z_{3} z_{5} t^{4}+x^{53} z_{1} z_{2} z_{3} z_{4} t^{4}+x^{34} z_{1} z_{5} z_{6} t^{3}+x^{35} z_{1} z_{4} z_{6} t^{3}+ \\
+x^{35} z_{1} z_{4} z_{5} t^{3}+x^{36} z_{1} z_{3} z_{6} t^{3}+x^{36} z_{1} z_{3} z_{5} t^{3}+x^{37} z_{1} z_{3} z_{4} t^{3} \\
+x^{47} z_{1} z_{2} z_{6} t^{3}+x^{47} z_{1} z_{2} z_{5} t^{3}+x^{48} z_{1} z_{2} z_{4} t^{3}+x^{49} z_{1} z_{2} z_{3} t^{3} \\
+x^{44} z_{1} z_{2} t^{2}+x^{33} z_{1} z_{3} t^{2}+x^{32} z_{1} z_{4} t^{2}+x^{31} z_{1} z_{5} t^{2}+x^{31} z_{1} z_{6} t^{2} .
\end{gathered}
$$

In a third step, we choose those terms that can not be divided by different ones, that is, we have the sum of the prime terms:

$$
x^{31} z_{2} z_{3} z_{4} z_{5} z_{6} t^{5}+x^{44} z_{1} z_{2} t^{2}+x^{33} z_{1} z_{3} t^{2}+x^{32} z_{1} z_{4} t^{2}+x^{31} z_{1} z_{5} t^{2}+x^{31} z_{1} z_{6} t^{2} .
$$

As the number of monomials in the later polynomial is 6 , it follows that, $\left|\mathcal{W}^{m}\right|=$ 6. To compute the Deegan-Packel index of a player $i \in N$ we should add those terms in which the variable $z_{i}$ is present, divided by the corresponding power of the variable $t$, and, finally, we should divide the obtained result by $\left|\mathcal{W}^{m}\right|$. For instance, to compute the Deegan-Packel index of player 4 we have that $z_{4}$ appears in two terms, and dividing by the corresponding power of variable $t$, we obtain $1 / 5+1 / 2=7 / 10$, and dividing again by $\left|\mathcal{W}^{m}\right|$ we get its Deegan-Packel index $7 / 60$.

We follow the same steps to compute the Deegan-Packel index for the other players:

$$
\rho(\mathcal{W})=\left(\frac{5}{12}, \frac{7}{60}, \frac{7}{60}, \frac{7}{60}, \frac{7}{60}, \frac{7}{60}\right) .
$$

In a similar way, we can compute the Public Good index from the last prime terms taking into account the definition

$$
h_{i}(\mathcal{W})=\frac{c_{i}(\mathcal{W})}{\sum_{j=1}^{n} c_{j}(\mathcal{W})} \text { so that } \sum_{i=1}^{n} h_{i}(\mathcal{W})=1,
$$

where $c_{i}(\mathcal{W})=\left|\mathcal{W}_{i}^{s}\right|=\left|\left\{S \in \mathcal{W}_{i}^{s} \wedge i \in S\right\}\right|$. In the case of Example 2 the last prime terms are

$$
x^{31} z_{2} z_{3} z_{4} z_{5} z_{6} t^{5}+x^{44} z_{1} z_{2} t^{2}+x^{33} z_{1} z_{3} t^{2}+x^{32} z_{1} z_{4} t^{2}+x^{31} z_{1} z_{5} t^{2}+x^{31} z_{1} z_{6} t^{2}
$$

and then we have

$$
h(\mathcal{W})=\frac{1}{15}(5,2,2,2,2,2)=\left(\frac{5}{15}, \frac{2}{15}, \frac{2}{15}, \frac{2}{15}, \frac{2}{15}, \frac{2}{15}\right) .
$$




\subsection{Shift power index and Shift Deegan-Packel power index}

Now we present a method to compute Shift and Shift Deegan-Packel power indices by means of the generating function in a weighted voting game.

As it happens with the Deegan-Packel index, we need to employ $n+2$ variables, one for each player, another to indicate the number of players in each coalition and, finally, a variable that indicates the weight of the coalition.

Let $(N, \mathcal{W})$ be a simple game, and let $s m_{k}^{i}(\mathcal{W})$ be the number of shift minimal winning coalitions of cardinality $k$ which the player $i$ belongs to. The shift power index of $i$ in that game is equal to

$$
f_{i}(\mathcal{W})=\frac{\sum_{k=1}^{n} s m_{k}^{i}(\mathcal{W})}{\sum_{j=1}^{n} \sum_{k=1}^{n} s m_{k}^{j}(\mathcal{W})}
$$

Now, the first step consists of computing the function $S\left(x, z_{1}, z_{2}, \ldots, z_{n}, t\right)$ described above. In the second step, we must remove such terms where the power of $x$ is less than $q$, that is, we choose only winning coalitions. We obtain a new function $S^{\prime}\left(x, z_{1}, z_{2}, \ldots, z_{n}, t\right)$.

After this second step, we define a partition $P=\left\{P_{1}, P_{2}, \ldots, P_{m}\right\}$ of the set of players $N$ in such a way that two players $i, j \in P_{r}$ if they belong to the same number of winning coalitions, that is, the number of terms of the function $S^{\prime}$ that contains to $z_{i}$ coincides with the number of terms of the function $S^{\prime}$ that contains to $z_{j}$. If $r<r^{\prime}$, and $i \in P_{r}$ and $j \in P_{r^{\prime}}$ the number of terms of $S^{\prime}$ that contains to $z_{i}$ is greater than the number of terms that contain to $z_{j}$. It is clear then, that $1 \in P_{1}$ and $n \in P_{m}$. In this third step, we remove from $S^{\prime}$ such terms that can be divided among a different term of the function. We obtain a new function $S^{\prime \prime}$. The total number of terms of this function coincides with the number of minimal winning coalitions of the game, $\left|\mathcal{W}^{m}\right|$. This function $S^{\prime \prime}$ can be written as:

$$
S^{\prime \prime}=S^{\prime \prime}(1)+S^{\prime \prime}(2)+S^{\prime \prime}(3)+\ldots+S^{\prime \prime}(n)
$$

where a term of $S^{\prime \prime}$ belongs to $S^{\prime \prime}(k)$ if the power of $t$ is equal to $k$, i.e., if the number of players involved in that term is $k$.

From $S^{\prime \prime}$ we remove the terms

$$
x^{a} \cdot z_{i_{1}} \cdot z_{i_{2}} \cdot \ldots \cdot z_{i_{j-1}} \cdot z_{i_{j}} \cdot z_{i_{j+1}} \cdot \ldots \cdot z_{i_{k-1}} \cdot z_{i_{k}} \cdot t^{k} \in S^{\prime \prime}(k)
$$

with $i_{j} \in P_{r}$, if there exists a term

$$
x^{a} \cdot z_{i_{1}} \cdot z_{i_{2}} \cdot \ldots \cdot z_{i_{j-1}} \cdot z_{i_{p}} \cdot z_{i_{j+1}} \cdot \ldots \cdot z_{i_{k-1}} \cdot z_{i_{k}} \cdot t^{k} \in S^{\prime \prime}(k)
$$

with $i_{p} \in P_{r^{\prime}}$ and $r<r^{\prime}$. After these deletions, we obtain a function $S^{\prime \prime \prime}$. The total number of terms of this new function $S^{\prime \prime \prime}$ coincides with the number of shift minimal winning coalitions of the game. 
Finally, on the one hand, to obtain the Shift power index of player $i \in N$ we only need to select those terms of the previous function $S^{\prime \prime \prime}$ in which the variable $z_{i}$ appears.

Example 3. Consider the weighted voting game with representation

$$
[5 ; 4,3,1,1,1] \text {. }
$$

In a first step, we compute the function $S\left(x, z_{1}, z_{2}, \ldots, z_{5}, t\right)$

$$
\begin{gathered}
S\left(x, z_{1}, z_{2}, \ldots, z_{5}, t\right)=\prod_{j=1}^{n}\left(1+x^{w_{j}} z_{j} t\right)= \\
\left(1+x^{4} z_{1} t\right)\left(1+x^{3} z_{2} t\right)\left(1+x z_{3} t\right)\left(1+x z_{4} t\right)\left(1+x z_{5} t\right)= \\
x^{10} z_{1} z_{2} z_{3} z_{4} z_{5} t^{5}+x^{6} z_{2} z_{3} z_{4} z_{5} t^{4}+x^{7} z_{1} z_{3} z_{4} z_{5} t^{4}+x^{9} z_{1} z_{2} z_{4} z_{5} t^{4}+ \\
x^{9} z_{1} z_{2} z_{3} z_{5} t^{4}+x^{9} z_{1} z_{2} z_{3} z_{4} t^{4}+x^{3} z_{3} z_{4} z_{5} t^{3}+x^{5} z_{2} z_{4} z_{5} t^{3}+ \\
x^{5} z_{2} z_{3} z_{5} t^{3}+x^{5} z_{2} z_{3} z_{4} t^{3}+x^{6} z_{1} z_{4} z_{5} t^{3}+x^{6} z_{1} z_{3} z_{5} t^{3}+x^{6} z_{1} z_{3} z_{4} t^{3}+ \\
+x^{8} z_{1} z_{2} z_{5} t^{3}+x^{8} z_{1} z_{2} z_{4} t^{3}+x^{8} z_{1} z_{2} z_{3} t^{3}+ \\
x^{7} z_{1} z_{2} t^{2}+x^{5} z_{1} z_{3} t^{2}+x^{5} z_{1} z_{4} t^{2}+x^{5} z_{1} z_{5} t^{2}+ \\
x^{4} z_{2} z_{3} t^{2}+x^{4} z_{2} z_{4} t^{2}+x^{4} z_{2} z_{5} t^{2}+x^{2} z_{3} z_{4} t^{2}+ \\
x^{2} z_{3} z_{5} t^{2}+x^{2} z_{4} z_{5} t^{2}+ \\
x^{4} z_{1} t+x^{3} z_{2} t+x z_{3} t+x z_{4} t+x z_{5} t+1
\end{gathered}
$$

In a second step, we choose those terms in which the power of $x$ is greater than or equal to 5 , that is,

$$
\begin{gathered}
S^{\prime}\left(x, z_{1}, z_{2}, \ldots, z_{5}, t\right)= \\
x^{10} z_{1} z_{2} z_{3} z_{4} z_{5} t^{5}+x^{6} z_{2} z_{3} z_{4} z_{5} t^{4}+x^{7} z_{1} z_{3} z_{4} z_{5} t^{4}+x^{9} z_{1} z_{2} z_{4} z_{5} t^{4}+ \\
x^{9} z_{1} z_{2} z_{3} z_{5} t^{4}+x^{9} z_{1} z_{2} z_{3} z_{4} t^{4}+x^{5} z_{2} z_{4} z_{5} t^{3}+ \\
x^{5} z_{2} z_{3} z_{5} t^{3}+x^{5} z_{2} z_{3} z_{4} t^{3}+x^{6} z_{1} z_{4} z_{5} t^{3}+x^{6} z_{1} z_{3} z_{5} t^{3}+x^{6} z_{1} z_{3} z_{4} t^{3}+ \\
+x^{8} z_{1} z_{2} z_{5} t^{3}+x^{8} z_{1} z_{2} z_{4} t^{3}+x^{8} z_{1} z_{2} z_{3} t^{3}+ \\
x^{7} z_{1} z_{2} t^{2}+x^{5} z_{1} z_{3} t^{2}+x^{5} z_{1} z_{4} t^{2}+x^{5} z_{1} z_{5} t^{2} .
\end{gathered}
$$

The partition defined by the previous function is $P=\{\{1\},\{2\},\{3,4,5\}\}$ because the respective number of monomials containing $i$ for $i=1,2,3,4,5$ is $15,12,11,11,11$.

In a third step, we choose those terms that can not be divided by different ones, that is, we have the function:

$$
\begin{gathered}
S^{\prime \prime}\left(x, z_{1}, z_{2}, \ldots, z_{5}, t\right)=x^{5} z_{2} z_{4} z_{5} t^{3}+x^{5} z_{2} z_{3} z_{5} t^{3}+x^{5} z_{2} z_{3} z_{4} t^{3}+ \\
x^{7} z_{1} z_{2} t^{2}+x^{5} z_{1} z_{3} t^{2}+x^{5} z_{1} z_{4} t^{2}+x^{5} z_{1} z_{5} t^{2}
\end{gathered}
$$

As the terms $x^{7} z_{1} z_{2} t^{2}, x^{5} z_{1} z_{3} t^{2} \in S^{\prime \prime}(2)$ and $2 \in P_{2}$ and $3 \in P_{3}$, we remove the term $x^{7} z_{1} z_{2} t^{2}$ in $S^{\prime \prime}$ to get $S^{\prime \prime \prime}$. To compute the shift power index of a player $i \in N$ we add those terms in $S^{\prime \prime \prime}$ in which the variable $z_{i}$ is present, that is: $s_{i}(\mathcal{W})=3$ for all $i \in N$, and therefore $f_{i}(\mathcal{W})=1 / 5$ for all $i \in N$. 
Analogously, for the Shift Deegan-Packel index we use $S^{\prime \prime \prime}$ and for each player $i \in N$ we add the converses of the power of $t$ for each monomial where $i$ is presented.

With this procedure, for Example 3 we obtain:

$$
\mu_{i}(\mathcal{W})=\left\{\begin{array}{cc}
\frac{1}{4} & \text { if } i=1 \\
\frac{1}{6} & \text { if } i=2 \\
\frac{7}{36} & \text { otherwise }
\end{array}\right.
$$

\section{Some comments on complexity}

The computation of Deegan-Packel, Public Good, Shift and Shift DeeganPackel power indices are based on function

$$
S\left(x, z_{1}, z_{2}, \ldots z_{n}, t\right)=\prod_{k=1}^{n}\left(1+x^{w_{k}} z_{k} t\right) .
$$

The number of products to compute $S\left(x, z_{1}, z_{2}, \ldots, z_{n}, t\right)$ is $\mathcal{O}\left(2^{n}\right)$. Thus, Deegan-Packel and Public Good power indices can be computed in $\mathcal{O}\left(2^{n}\right)$, whereas Shift and Shift Deegan-Packel power indices require $\mathcal{O}\left(2^{n} n\right)$ because it also needs to compute $S^{\prime \prime}$.

However, when computing $S\left(x, z_{1}, z_{2}, \ldots, z_{n}, t\right)$ we could stop the products among monomials when the power $\alpha$ of $x$ verifies that $\alpha \geq q$. That is, we could directly compute only the sum of the weights of the players that belong to $S \in\left(\mathcal{L} \cup \mathcal{W}^{m}\right)$. Thus, the complexity to compute Deegan-Packel and Public Good power indices is

$$
\mathcal{O}\left(\sum_{S \in\left(\mathcal{L} \cup \mathcal{W}^{m}\right)} s\right)
$$

whereas the complexity to compute Shift and Shift Deegan-Packel power indices is

$$
\mathcal{O}\left(n \sum_{S \in\left(\mathcal{L} \cup \mathcal{W}^{m}\right)} s\right) \text {. }
$$

Note that, although the time complexity is high (not polinomial, in general) as in other methods, here we have an advantage with the space complexity required because generating funtions need a minimum space to do sums and products. Moreover, it is easier to compute power indices with generating functions than using other methods from the definition; see [37] to know other methods to compute power indices.

\section{Conclusions and Future Work}

We present some known indices for simple games and some methods to compute them. As far as we know, we define a new power index, the Shift 
Deegan-Packel power index, which combines the ideas of the Shift power index and the Deegan-Packel power index. We describe a new method to compute four power indices (Deegan-Packel, Public Good, Shift and Shift Deegan-Packel) by means of generating functions. The corresponding (exponential) complexity depends on losing coalitions plus minimal winning coalitions.

For future work, it would be interesting to reduce the complexity with similar techniques by means of generating functions, and to compare our techniques with others like (Quasi-reduced and Ordered) Binary Decision Diagrams [8] or polinomial algorithms [5].

\section{Acknowledgements}

The authors are grateful to the anonymous reviewers of this article for making helpful suggestions that allowed to improve this final version.

\section{References}

[1] J.M. Alonso-Meijide, J.M. Bilbao, B. Casas-Méndez, and J.R. Fernández. Weighted multiple majority games with unions: Generating functions and applications to the European Union. European Journal of Operational Research, 198:530-544, 2009.

[2] J.M. Alonso-Meijide and C. Bowles. Generating Functions for Coalitional Power Indices: An Application to the IMF. Annals of Operations Research, 137:21-44, 2005.

[3] J.M. Alonso-Meijide, B. Casas-Méndez, M.J. Holler, and S. Lorenzo-Freire. Computing power indices: Multilinear extensions and new characterizations. European Journal of Operational Research, 188:540-554, 2008.

[4] J.M. Alonso-Meijide and J. Freixas. A new power index based on minimal winning coalitions without any surplus. Decision Support Systems, 49:7076,2010 .

[5] H. Aziz. Algorithmic and complexity aspects of simple coalitional games. $\mathrm{PhD}$ thesis, University of Warwick, 2009.

[6] J.F. Banzhaf. Weighted voting doesn't work: A mathematical analysis. Rutgers Law Review, 19:317-343, 1965.

[7] J.M. Bilbao, J. R. Fernández, A. Jiménez Losada, and J. J. López. Generating functions for computing power indices efficiently. TOP, 8:191-213, 2000 .

[8] S. Bolus. Power indices of simple games and vector-weighted majority games by means of binary decision diagrams. European Journal of Operational Research, 210:258-272, 2011. 
[9] S.J. Brams and P.J. Affuso. Power and size: A new paradox. Theory and Decision, 7:29-56, 1976.

[10] D.G. Cantor. On the ambiguity of backus systems. Journal of the ACM, 9:477-479, 1962.

[11] J.A. De Loera, R. Hemmecke, and M. Köppe. Pareto optima of multicriteria integer linear programs. Informs Journal on Computing, 21:39-48, 2009.

[12] J. Deegan and E.W. Packel. A new index of power for simple $n$-person games. International Journal of Game Theory, 7:113-123, 1979.

[13] P. Flajolet and R. Sedgewick. An Introduction to the Analysis of Algorithms. Addison-Wesley, 1996.

[14] J. Freixas. Power indices. Wiley Encyclopedia of Operations Research and Management Science, 2011. 19 pages. http://onlinelibrary.wiley.com/doi/10.1002/9780470400531.eorms0669/pdf

[15] M.J. Holler. Forming coalitions and measuring voting power. Political Studies, 30:262-271, 1982.

[16] J.R. Isbell. A class of simple games. Duke Mathematics Journal, 25:423439, 1958.

[17] K. Ishikawa. Fundamentals of simple games from a viewpoint of blockability relations. Applied Mathematics and Computation, 211:45-53, 2009.

[18] M. Iwase and K. Ohno. The performance evaluation of a multi-stage jit production system with stochastic demand and production capacities. European Journal of Operational Research, 214:216-222, 2011.

[19] R.J. Johnston. On the measurement of power: Some reactions to Laver. Environment and Planning A, 10:907-914, 1978.

[20] K. Kojima and T. Inohar. Methods for comparison of coalition influence on games in characteristic function form and their interrelationships. Applied Mathematics and Computation, 217:4047-4050, 2010.

[21] D. Leech. Computation of power indices. The Warwick Economics Research Paper Series (TWERPS), 644, 2002.

[22] G. Levitin. Asymmetric weighted voting systems. Reliability Engineering E System Safety, 76:199-206, 2002.

[23] G. Levitin. Evaluating correct classification probability for weighted voting classifiers with plurality voting. European Journal of Operational Research, 141:596-607, 2002.

[24] G. Levitin. Optimal allocation of multi-state elements in linear consecutively connected systems with vulnerable nodes. European Journal of Operational Research, 150:406-419, 2003. 
[25] G. Levitin and A. Lisniansk. Reliability optimization for weighted voting system. Reliability Engineering $\mathcal{E} 3$ System Safety, 71:131-138, 2001.

[26] G. Levitin. Threshold optimization for weighted voting classifiers. Naval Research Logistics, 50:322-344, 2003.

[27] G. Levitin. Uneven allocation of elements in linear multi-state sliding window system. European Journal of Operational Research, 163:418-433, 2005.

[28] G. Levitin. Weighted voting systems: reliability versus rapidity. Reliability Engineering \&3 System Safety, 89:177-184, 2005.

[29] W.F. Lucas. Measuring power in weighted voting system. S. Brams (Ed.), Political and Related Models, Springer-Verlag, 1983.

[30] A. Nijenhuis and H.S. Wilf. Combinatorial Algorithms: For Computers and Calculators. Academic Press, Inc., 1978.

[31] G. Owen. Multilinear extensions of games. Management Science, 18:64-79, 1972.

[32] G. Owen. Multilinear extensions and the Banzhaf value. Naval Res. Logist. Quart., 22:741-750, 1975.

[33] L.S. Shapley. A value for $n$-person games. Contributions to the Theory of Games. Annals of Mathematical Studies, 28, Princeton: Princeton University Press, 2:307-317, 1953.

[34] L.S. Shapley and M. Shubik. A method for evaluating the distribution of power in a committee system. American Political Science Review, 48:787$792,1954$.

[35] A.D. Taylor and W.S. Zwicker. Simple games: desirability relations, trading, and pseudoweightings. Princeton University Press, New Jersey, USA, 1999.

[36] I. Ushakov. The method of generalized generating sequences. European Journal of Operational Research, 125:316-323, 2000.

[37] Web-based Algorithms for Voting Power Analysis. URL: http://homepages.warwick.ac.uk/ẽcaae/.

[38] H.S. Wilf. Generatingfunctionology. Academic Press, Inc., 1990.

[39] A. Yamazaki, T. Inohara, and B. Nakano. New interpretation of the core of simple games in terms of voters' permission. Applied Mathematics and Computation, 108:115-127, 2000.

[40] E. Zio and R. Bazzo. A clustering procedure for reducing the number of representative solutions in the Pareto front of multiobjective optimization problems. European Journal of Operational Research, 210:624-634, 2011. 